\title{
ALMOST ASSOCIATIVE OPERATIONS GENERATING A MINIMAL CLONE
}

\author{
TAMÁS WALDHAUSER
}

\begin{abstract}
Characterizations of 'almost associative' binary operations generating a minimal clone are given for two interpretations of the term 'almost associative'. One of them uses the associative spectrum, the other one uses the index of nonassociativity to measure how far an operation is from being associative.
\end{abstract}

\section{Minimal Clones}

A clone on a set $A$ is a set of finitary operations on $A$ that is closed under composition of functions and contains all the projections. The base set $A$ can be arbitrary; we will never assume finiteness in this paper. If $\mathbb{A}=(A ; F)$ is an algebra, then the set of term functions, denoted by $C l o(\mathbb{A})$, is a clone on $A$, the clone of the algebra $\mathbb{A}$. In this case $C l o(\mathbb{A})$ is the smallest clone containing $F$, therefore we say that $F$ generates the clone, and we write $[F]=C l o(\mathbb{A})$. (Clearly, every clone arises as the clone of an algebra: we just need to pick a generating set for the clone, and let these be the basic operations of the algebra.) We can also speak about $C l o(\mathcal{V})$, the clone of a variety $\mathcal{V}$. By this we mean the clone of $\mathbb{F}_{\aleph_{0}}(\mathcal{V})$, the countably generated free algebra of $\mathcal{V}$.

The $n$-ary part of $C l o(\mathbb{A})$, denoted by $C l o^{(n)}(\mathbb{A})$ can be identified naturally with $\mathbb{F}_{n}(V(\mathbb{A}))$, the $n$-generated free algebra of the variety generated by $\mathbb{A}$. Projections correspond to variables under this identification: the first binary projection is $e_{1}$ : $(x, y) \mapsto x$ and the second one is $e_{2}:(x, y) \mapsto y$, therefore we will sometimes think of the variables $x$ and $y$ as projections (most of the time we will work with binary operations).

All clones on a given set $A$ form a lattice with respect to inclusion; the smallest element of this lattice is the trivial clone, the clone of all projections on $A$, while the greatest element is the clone of all finitary operations on A. Minimal clones are the atoms of this lattice, i.e. a clone is minimal, if its only proper subclone is the trivial clone. A minimal clone is generated by any of its nontrivial (i.e. non-projection) elements, thus all minimal clones are singly generated, and therefore arise as clones of algebras with just one basic operation. If $\mathbb{A}=(A ; f)$ is such an algebra, then in order to prove that it has a minimal clone, one needs to verify that $f \in[g]$ holds for every nontrivial $g \in C l o(\mathbb{A})$. This fact can be expressed by identities, so if $\mathbb{A}$ has a minimal clone, then so does $V(\mathbb{A})$, and if a variety $\mathcal{V}$ has a minimal clone, then the clone of any algebra in $\mathcal{V}$ is either minimal or trivial.

To prove that a clone $[f]$ on $A$ is not minimal one needs to find a nontrivial operation $g \in[f]$ such that $f \notin[g]$. This can be done for example by showing that there is an equivalence relation $\rho$ on $A$ (a subset of $A$ ), such that $\rho$ is a congruence (subuniverse) of the algebra $(A ; g)$, but is not a congruence (subuniverse) of $(A ; f)$. (There is a general notion of preservation of relations of arbitrary arity, and this gives a Galois-correspondence between operations and relations on finite sets 1, 8,

2000 Mathematics Subject Classification. 08A40, 20 N02.

Key words and phrases. Clone, minimal clone, groupoid, associativity.

Research supported by the Hungarian National Foundation for Scientific Research grant no. T 048809 . 
but we will use only the previous observation, which is valid for infinite sets as well.)

It is convenient to generate a minimal clone by a nontrivial operation of the smallest arity. Minimal clones are classified with respect to this generator; there are five types, and for two of them there is a complete characterization of minimal clones (Rosenberg's Theorem, see [25] and 29]).

One of the three types where the description of minimal clones is not complete yet is the binary case. Clones of this type are generated by an idempotent binary operation, so they can be (and will be) viewed as clones of idempotent groupoids. (In this paper the term groupoid refers to an algebra with a single binary operation.) The basic operation of a groupoid will be denoted by $f(x, y)=x y$, and by the dual of $\mathbb{A}=(A ; f)$ we mean the groupoid $\mathbb{A}^{d}=\left(A ; f^{d}\right)$ with $f^{d}(x, y)=f(y, x)=$ $y x$. Similarly, $\mathcal{V}^{d}$ denotes the variety formed by the duals of the elements of $\mathcal{V}$. Obviously, a groupoid has a minimal clone if and only if its dual does (actually they have the very same clone).

A groupoid has a trivial clone if and only if it is a left or right zero semigroup. The simplest examples of groupoids (or varieties) with a minimal clone are semilattices and rectangular bands. Before giving more examples of varieties with a minimal clone, let us make some notational conventions.

To save parentheses we use the notation $\overleftarrow{x_{1} \cdot \ldots \cdot x_{n}}$ for the left-associated product $\left(\cdots\left(\left(x_{1} x_{2}\right) x_{3}\right) \cdots\right) x_{n}$, and similarly $\overrightarrow{x_{1} \cdot \ldots \cdot x_{n}}$ for the right-associated product $x_{1}\left(\cdots\left(x_{n-2}\left(x_{n-1} x_{n}\right)\right) \cdots\right)$. We abbreviate $\overleftarrow{x \cdot y \cdot \ldots \cdot y}$ to $x y^{n}$ (where $n$ is certainly the number of $y$ 's appearing in the product). Analogously ${ }^{n} x y$ stands for $\overrightarrow{x \cdot \ldots \cdot x \cdot y}$.

Let $\mathcal{B}$ denote the variety defined by the identities $x x \approx x, x(x y) \approx x(y x) \approx$ $(x y) x \approx(x y) y \approx(x y)(y x) \approx x y$; let $\mathcal{C}_{p}$ be the variety of $p$-cyclic groupoids (cf. [23]) defined by $x x \approx x, x(y z) \approx x y,(x y) z \approx(x z) y, x y^{p} \approx x$, and finally, let $\mathcal{D}$ be defined by $x(y x) \approx(x y) x \approx(x y) y \approx(x y)(y x) \approx x y$ and $x \cdot \overleftarrow{x \cdot y_{1} \cdot \ldots \cdot y_{n}} \approx x$ (for all $n \geq 0$ ). The clone of $\mathcal{B}$ and $\mathcal{D}$ is minimal, while the clone of $\mathcal{C}_{p}$ is minimal iff $p$ is a prime. The minimality of the clone of $\mathcal{B}$ and $\mathcal{D}$ is proved in [20]; these are the clones in parts (c) and (d) in Theorem 5.2. For the proof of the minimality of the clones of $p$-cyclic groupoids see [22]. (From now on we always assume that $p$ is a prime number, when we speak about $p$-cyclic groupoids.)

The following propositions show the usefulness of absorption identities in the study of minimal clones. These are identities of the form $t \approx x$, i.e. identities with a single variable on one side. The proofs of these propositions can be found in [20] and 12 .

Proposition 1.1. Let $\mathcal{V}$ be a variety with a minimal clone, and let $\mathbb{A} \in \mathcal{V}$ have a nontrivial (hence minimal) clone. Then $\mathcal{V}$ satisfies every absorption identity that holds in $\mathbb{A}$.

Proof. See Lemma 2.1 in [20] or Lemma 3.6 in [12].

Proposition 1.2. Let $\mathcal{V}$ be a variety with a minimal clone, and suppose that $\mathcal{V}$ contains a p-cyclic groupoid (rectangular band) with a nontrivial clone. Then $\mathcal{V}$ is the variety of p-cyclic groupoids (rectangular bands).

Proof. By the previous proposition, it suffices to show that $p$-cyclic groupoids and rectangular bands are axiomatizable by absorption identities. For $p$-cyclic groupoids such an axiomatization is given in Lemma 3.10 of [12, and the method described in Lemma 2.3 of 20 yields (almost) the same identities. For rectangular bands see Lemma 3.8 of [12] or Theorem 5.2 (b) of [20] for a list of absorption identities. Note that the conclusion of the proposition says that " $\mathcal{V}$ is the variety of $p$-cyclic groupoids (rectangular bands)", not that " $\mathcal{V}$ is $a$ variety of $p$-cyclic groupoids (rectangular bands)". This is because the only nontrivial subvariety of $\mathcal{C}_{p}$ is the variety of left zero semigroups (see the last paragraph of the proof of Lemma 3.5 in [12] or 
Corollary 2.1 in [20]), and clearly the variety of rectangular bands does not have a proper subvariety with a nontrivial clone either.

Proposition 1.3. Let $\mathcal{V}$ be a variety with a minimal clone satisfying the identities $x x \approx x, x(y x) \approx(x y) x \approx(x y) y \approx(x y)(y x) \approx x y, x(x y) \approx x$. Then $\mathcal{V}$ is $a$ subvariety of $\mathcal{D}$.

Proof. This is part (d) of Theorem 5.2 in 20 . The identities listed here are sufficient to determine the two-generated free algebra of $\mathcal{V}$. Its multiplication table is the following (the four elements have to be distinct, since otherwise $C l o(\mathcal{V})$ would be trivial).

\begin{tabular}{l|llll}
$\cdot$ & $x$ & $y$ & $x y$ & $y x$ \\
\hline$x$ & $x$ & $x y$ & $x$ & $x y$ \\
$y$ & $y x$ & $y$ & $y x$ & $y$ \\
$x y$ & $x y$ & $x y$ & $x y$ & $x y$ \\
$y x$ & $y x$ & $y x$ & $y x$ & $y x$
\end{tabular}

It is not hard to check that this groupoid satisfies every identity of the form $x \cdot \overleftarrow{x \cdot y_{1} \cdot \ldots \cdot y_{n}} \approx x$ (this is a special case of Lemma 4.2 in 20]). These are absorption identities, therefore we can apply Proposition 1.1 with $\mathbb{A}=\mathbb{F}_{2}(\mathcal{V})$ to show that $\mathcal{V}$ satisfies these identities, too. The remaining identities in the definition of $\mathcal{D}$ are the same as that were assumed.

Characterizing minimal clones in general is a hard task, even in the binary case. All known results describe minimal clones under certain restrictions [4, 5, 11, 12, 13, 20, 24, 28, 31, 32. Another result of this kind is the description of associative binary operations generating a minimal clone [21, 30]: a semigroup has a minimal clone iff it is a rectangular band, a left regular band (idempotent semigroup satisfying $x y x \approx x y$ ) or a right regular band (dual of a left regular band). Note that left and right regular bands belong to the varieties $\mathcal{B}$ and $\mathcal{B}^{d}$, respectively. In this paper we slightly generalize this result by characterizing 'almost associative' binary operations generating a minimal clone. To explain what we mean by being 'almost associative', we need a way to measure how far a certain operation is from being associative. We discuss two such measures: the associative spectrum and the index of nonassociativity. In Section 2 we characterize groupoids with a minimal clone and small associative spectrum (Theorem 2.8), and in Section 3 we describe groupoids with a minimal clone and small index of nonassociativity (Theorem 3.3.

\section{Minimal Clones With SMALl ASsociative SPeCtRum}

One way of measuring associativity is possible by considering the identities implied by associativity, and somehow counting how many of these are (not) satisfied. To make this more precise, let us say that $B$ is a bracketing, if $B$ is a groupoid term, and each variable occurs exactly once in $B$. If these variables are $x_{1}, x_{2}, \ldots, x_{n}$ and they appear in this order (as we will suppose most of the time), then $B$ is nothing else but a way to put brackets into the product $x_{1} \cdot \ldots \cdot x_{n}$ such that the order of the $n-1$ multiplications is well determined. In this case we say that $B$ is a bracketing of the product $x_{1} \cdot \ldots \cdot x_{n}$, and we write $B=B\left(x_{1}, \ldots, x_{n}\right)$. The number of variables appearing in $B$ is called the size of $B$, and is denoted by $|B|$.

In every bracketing there is an outermost multiplication, and this splits the bracketing into two parts, the left factor and the right factor of the bracketing. Let $B=B\left(x_{1}, \ldots, x_{n}\right)$, and let $P, Q$ be the left and right factors of $B$. Then $B=P Q$, and $P=P\left(x_{1}, \ldots, x_{k}\right), Q=Q\left(x_{k+1}, \ldots, x_{n}\right)$, where $k=|P|$. Sometimes we will use the notation $l(B)$ for the left factor of $B$.

The number of bracketings of the product $x_{1} \cdot \ldots \cdot x_{n}$ is $C_{n-1}=\frac{1}{n}\left(\begin{array}{c}2 n-2 \\ n-1\end{array}\right)$, the $(n-1)$ st Catalan number. In a semigroup, all of these $C_{n-1}$ many terms induce the same term function, but in an arbitrary groupoid they may induce more than one term function. Intuitively, the more term functions of this kind there are, the 
less associative the multiplication is. Therefore we define the associative spectrum of a groupoid $\mathbb{A}$ to be the sequence $s_{\mathbb{A}}(1), s_{\mathbb{A}}(2), \ldots, s_{\mathbb{A}}(n), \ldots$, where $s_{\mathbb{A}}(n)$ is the number of different term functions on $\mathbb{A}$ arising from bracketings of $x_{1} \ldots \ldots$. $x_{n}$. Thus the associative spectrum gives (only quantitative) information about identities of the form $B_{1}\left(x_{1}, \ldots, x_{n}\right) \approx B_{2}\left(x_{1}, \ldots, x_{n}\right)$ satisfied by the groupoid. The associative spectrum was introduced and investigated in 6 .

Clearly, $s_{\mathbb{A}}(1)=s_{\mathbb{A}}(2)=1$ for every groupoid $\mathbb{A}$, and $s_{\mathbb{A}}(3)=1$ iff $\mathbb{A}$ is a semigroup. In the latter case $s_{\mathbb{A}}(n)=1$ for all $n$ by the general law of associativity. The smallest possible spectrum for a nonassociative multiplication is $1,1,2,1,1, \ldots$, so we could say that a binary operation is almost associative, if its spectrum is this sequence. However, there is no groupoid having a minimal clone with this spectrum (not even an idempotent groupoid) as we will see later. Therefore we have to be more generous: in Theorem 2.8 we determine groupoids with a minimal clone satisfying $s(4)<5=C_{3}$. First we prove three theorems which show that certain identities of the form $B_{1}\left(x_{1}, \ldots, x_{n}\right) \approx B_{2}\left(x_{1}, \ldots, x_{n}\right)$ cannot hold in nonassociative groupoids with a minimal clone, and then we discuss the four-variable case in detail. (In the first two theorems we actually assume only idempotence.)

Theorem 2.1. If an idempotent groupoid satisfies the identity

$$
\overleftarrow{x_{1} \cdot \ldots \cdot x_{n}} \approx \overline{x_{1} \cdot \ldots \cdot x_{n}}
$$

for some $n \geq 3$, then it is a semigroup.

Proof. Applying 2.1 with $x_{1}=\ldots=x_{k}=x, x_{k+1}=\ldots=x_{n}=y$ we obtain

$$
x y^{n-k} \approx \overleftarrow{x \cdot \ldots \cdot x \cdot y \cdot \ldots \cdot y} \approx \overline{x \cdot \ldots \cdot x \cdot y \cdot \ldots \cdot y} \approx{ }^{k} x y
$$

for $1 \leq k \leq n-1$.

Let us use (2.1) again, for $x_{1}=x, x_{2}=u=x y^{2} \approx{ }^{n-2} x y, x_{3}=\ldots=x_{n}=y$ :

$$
(x u) y^{n-2} \approx \overleftarrow{x \cdot u \cdot y \cdot \ldots \cdot y} \approx \overline{x \cdot u \cdot y \cdot \ldots \cdot y} \approx x(u y)
$$

The left hand side is $(x u) y^{n-2} \approx\left({ }^{n-1} x y\right) y^{n-2} \approx(x y) y^{n-2} \approx x y^{n-1} \approx x y$ (we used (2.2) twice, with $k=n-1$ and $k=1$ respectively). We can compute the right hand side of 2.3 in a similar manner: $x(u y) \approx x\left(x y^{3}\right) \approx x\left({ }^{n-3} x y\right) \approx{ }^{n-2} x y \approx$ $x y^{2}$. Thus we have $x y \approx x y^{2}$, i.e. right multiplications are idempotent.

Finally, to prove associativity, we write up 2.1 one more time:

$$
(x y) z^{n-2} \approx \overleftarrow{x \cdot y \cdot z \cdot \ldots \cdot z} \approx \overline{x \cdot y \cdot z \cdot \ldots \cdot z} \approx x(y z) .
$$

By the idempotence of right multiplication (by $z$ ) the left hand side reduces to $(x y) z$, and therefore associativity is established.

Theorem 2.2. An idempotent groupoid satisfying the following two identities for some $n \geq 3$, must be a semigroup.

$$
\begin{aligned}
& x_{0} \cdot \overleftarrow{x_{1} \cdot \ldots \cdot x_{n}} \approx x_{0} \cdot \overrightarrow{x_{1} \cdot \ldots \cdot x_{n}} \\
& \overleftarrow{x_{1} \cdot \ldots \cdot x_{n}} \cdot x_{0} \approx \overline{x_{1} \cdot \ldots \cdot x_{n}} \cdot x_{0}
\end{aligned}
$$

Proof. Substituting $\overleftarrow{x_{1} \cdot \ldots \cdot x_{n}}$ into $x_{0}$ in the first identity we have

$$
\overleftarrow{x_{1} \cdot \ldots \cdot x_{n}} \approx \overleftarrow{x_{1} \cdot \ldots \cdot x_{n}} \cdot \overline{x_{1} \cdot \ldots \cdot x_{n}}
$$

by idempotence. Similarly, if we substitute $\overrightarrow{x_{1} \cdot \ldots \cdot x_{n}}$ for $x_{0}$ in the second identity, then we get

$$
\overleftrightarrow{x_{1} \cdot \ldots \cdot x_{n}} \cdot \overrightarrow{x_{1} \cdot \ldots \cdot x_{n}} \approx \overrightarrow{x_{1} \cdot \ldots \cdot x_{n}}
$$

and thus (2.1), hence also associativity follows by the previous theorem.

Theorem 2.3. If a groupoid has a minimal clone and satisfies

$$
\overleftarrow{x_{1} \cdot \ldots \cdot x_{n}} \approx x_{1} \cdot \overleftarrow{x_{2} \cdot \ldots \cdot x_{n}}
$$

for some $n \geq 3$, then it is a semigroup. 
Proof. The case $n=3$ is trivial, so let us suppose that $n \geq 4$. First we draw a consequence of (2.4) and idempotence (putting $x$ and $z$ for $x_{1}$ and $x_{n}$, and $y$ for the rest of the variables):

$$
\left(x y^{n-2}\right) z \approx x(y z) .
$$

As a special case (with $z=y$ ) we get

$$
x y^{n-1} \approx x y .
$$

Now we suppose that $\mathbb{A}=(A ; \cdot)$ is a groupoid with a minimal clone that satisfies identity 2.4 . The binary operation $s(x, y)=x y^{n-2}$ belongs to the clone of $\mathbb{A}$, therefore if it is nontrivial, then $[s]$ contains the basic operation $f(x, y)=x y$.

Suppose that $a$ and $b$ are arbitrary elements of $A$ such that $c=(a b) a^{n-3} \neq a$. We claim that $s$ is a semilattice operation on the two-element set $\{a, c\}$. With the help of (2.6) we see that $s(c, a)=\left((a b) a^{n-3}\right) a^{n-2}=(a b) a^{2 n-5}=\left((a b) a^{n-1}\right) a^{n-4}=$ $((a b) a) a^{n-4}=(a b) a^{n-3}=c$. To compute $s(a, c)$ let us first consider $a c$ :

$$
a c=a\left((a b) a^{n-3}\right)=((a a) b) a^{n-3}=(a b) a^{n-3}=c .
$$

In the middle two steps we used identity (2.4) and idempotence. Now it is easy to conclude that $s(a, c)=a c^{n-2}=c$, proving that $s$ is indeed a semilattice operation on $\{a, c\}$.

Since $f \in[s]$, the restriction of $f$ to $\{a, c\}$ is either trivial, or coincides with $s$. In the latter case we have $f(c, a)=c$, so

$$
\left((a b) a^{n-3}\right) a=(a b) a^{n-2}=(a b) a^{n-3} .
$$

If $f$ is trivial on our two-element set, then it has to be a second projection, because $f(a, c)=a c=c$ as we have already observed in (2.7). Thus we have $f(c, a)=c a=a$, which means that $(a b) a^{n-2}=a$. Multiplying by $a$ from the right we get $(a b) a^{n-1}=a$, therefore $(a b) a=a$ by 2.6 . If we multiply both sides of this equality $n-4$ times by $a$, then we get $(a b) a^{n-3}=a$, i.e. $c=a$, contrary to our assumption.

If $(a b) a^{n-3}=a$ holds for $a, b \in A$, then 2.8 ) holds trivially. Thus we have proved that if a groupoid $\mathbb{A}$ has a minimal clone, and satisfies (2.4), then (2.8) holds for all $a, b \in A$. In other words, $\mathbb{A}$ satisfies the following identity.

$$
(x y) x^{n-3} \approx(x y) x^{n-2}
$$

It suffices to show now that (2.4) and (2.9) together with idempotence imply associativity. Let us multiply both sides of $(2.9)$ by $x$ from the right. We get $(x y) x^{n-2} \approx(x y) x^{n-1}$ and then $(2.6)$ shows that $(x y) x^{n-2} \approx(x y) x$. Therefore $\left((x y) x^{n-2}\right) z \approx((x y) x) z$ also holds. The left hand side of this identity reduces to $(x y)(x z)$ according to 2.5 , with $x y, x$ and $z$ playing the role of $x, y$ and $z$, respectively. Thus we have obtained the following identity.

$$
((x y) x) z \approx(x y)(x z)
$$

Now we go back to (2.9), and this time we multiply it by $y$ from the left. The left hand side becomes $y\left((x y) x^{n-3}\right)$, which turns to $((y x) y) x^{n-3}$ if we apply 2.4. With the help of 2.10$)$ and idempotence we can simplify this expression: $((y x) y) x^{n-3} \approx(((y x) y) x) x^{n-4} \approx((y x)(y x)) x^{n-4} \approx(y x) x^{n-4} \approx y x^{n-3}$. The right hand side of 2.9 becomes $y\left((x y) x^{n-2}\right)$. This can be considered as a product of $n$ factors, if we keep the $x$ and the $y$ in the middle together. We can rearrange this product according to (2.4), and we get $(y(x y)) x^{n-2}$. The $y(x y)$ at the beginning of this term can be written as $y \cdot \overleftarrow{x \cdot \ldots \cdot x \cdot y}$, and an application of 2.4 yields $\overleftarrow{y \cdot x \cdot \ldots \cdot x \cdot y} \approx\left(y x^{n-2}\right) y$. Substituting this back into the original expression we get $(y(x y)) x^{n-2} \approx\left(\left(y x^{n-2}\right) y\right) x^{n-2}$. If we consider $y x^{n-2}$ as one factor, then this is again a (left-associated) product of $n$ factors, and we can use (2.4) one more time: $\left(\left(y x^{n-2}\right) y\right) x^{n-2} \approx\left(y x^{n-2}\right)\left(y x^{n-2}\right)$. Clearly this is just $y x^{n-2}$, and if we 
compare the results we have obtained from the two sides of $(2.9)$ we can conclude the following identity.

$$
y x^{n-3} \approx y x^{n-2}
$$

Multiplying this by $x$ we get $y x^{n-2} \approx y x^{n-1} \approx y x$ by $(2.6)$. Now the left hand side of 2.5 can be simplified as $\left(x y^{n-2}\right) z \approx(x y) z$, and therefore associativity follows.

Remark. Idempotence and identity (2.4) for $n \geq 4$ do not imply associativity, as we can see from the following example. For every $k \geq 2$ we define a groupoid $\mathbb{A}_{k}$ on the set $A_{k}=\mathbb{Z}_{k} \dot{\cup}\{e\}$ by

$$
x y= \begin{cases}y & \text { if } y \neq e \\ x+1 & \text { if } y=e \neq x \\ e & \text { if } y=e=x .\end{cases}
$$

This groupoid is idempotent, but not associative, because $(0 e) e=2 \neq 1=0$ (ee). Let $B\left(x_{1}, \ldots, x_{n}\right)$ be a bracketing, and let $l_{i}$ denote the left depth of $x_{i}$ in $B$ (see 6 for the definition of left depth). It is not hard to prove by induction on $n$, that for any $c_{1}, \ldots, c_{n} \in A_{k}$ we have $B\left(c_{1}, \ldots, c_{n}\right)=c_{i}+l_{i}$ if $c_{i}$ is the last element of the sequence $c_{1}, \ldots, c_{n}$ that is different from $e$ (if there is no such element, then clearly $\left.B\left(c_{1}, \ldots, c_{n}\right)=e\right)$. Thus two bracketings give the same term function on $\mathbb{A}_{k}$ iff their left depth sequences are congruent modulo $k$. The left depth sequence of the bracketing on the left hand side of (2.4) is $(n-1, n-2, n-3, \ldots, 1,0)$ and that of the right hand side is $(1, n-2, n-3, \ldots, 1,0)$. Hence $\mathbb{A}_{k}$ satisfies 2.4 iff $k$ divides $n-2$. For example, $\mathbb{A}_{n-2}$ is an idempotent nonassociative groupoid satisfying (2.4).

The associative spectrum of $\mathbb{A}_{k}$ is the same as that of the operation $x+\varepsilon y$ on $\mathbb{C}$, where $\varepsilon$ is a primitive $k$-th root of unity: both count the number of $z a g$ sequences modulo $k$ (cf. [6] 2.8. and 6.4.). If $k=2$, then we have $\varepsilon=-1$, and the spectrum is $2^{n-2}$ (cf. 6] 3.1.). For $k=3$ the spectrum is sequence A005773 in the Encyclopedia 26]; this sequence is related to Motzkin numbers (A001006). The spectrum for $k=4$ does not appear in the Encyclopedia, but the superseeker found that it is a transform of the sequence A036765

Let us now turn to the investigation of four-variable 'associativity conditions'. There are five bracketings of size four:

$$
\begin{aligned}
& B_{1}=x(y(z u)) ; \\
& B_{2}=x((y z) u) ; \\
& B_{3}=(x y)(z u) ; \\
& B_{4}=((x y) z) u ; \\
& B_{5}=(x(y z)) u .
\end{aligned}
$$

Many of the possible $\left(\begin{array}{l}5 \\ 2\end{array}\right)$ identities cannot be satisfied by a nonassociative idempotent groupoid. For example, identifying $z$ and $u$ in $B_{1}$ and $B_{3}$ we see that $B_{1} \approx B_{3}$ implies associativity if idempotence is assumed. A similar argument works for $B_{3} \approx B_{4}$ and $B_{2} \approx B_{5}$. For $B_{2} \approx B_{3}$ we need two steps: multiplying both sides by a variable from the left yields $x(y((z u) v)) \approx x((y z)(u v))$ (after renaming the variables), while replacing $u$ with $u v$ gives $x((y z)(u v)) \approx(x y)(z(u v))$. Now $x(y((z u) v)) \approx(x y)(z(u v))$ follows by transitivity, and identifying $z, u$ and $v$ we get $x(y z) \approx(x y) z$. We can treat $B_{3} \approx B_{5}$ similarly (this is actually the dual of $\left.B_{2} \approx B_{3}\right)$.

Specializing Theorems 2.1 and 2.3 to $n=4$ we see that $B_{1} \approx B_{4}$ and $B_{2} \approx B_{4}$ cannot hold in a nonassociative groupoid with a minimal clone, and neither can $B_{1} \approx B_{5}$, because it is the dual of $B_{2} \approx B_{4}$. Only three possibilities remain: our groupoid satisfies $B_{1} \approx B_{2}$ or $B_{4} \approx B_{5}$ or both. Theorem 2.2 shows that the third case is impossible, hence we can conclude that if a groupoid $\mathbb{A}$ has a minimal clone, and $1<s_{\mathbb{A}}(4)<5$ holds for its spectrum, then $s_{\mathbb{A}}(4)=4$, and $\mathbb{A}$ satisfies either 
$B_{1} \approx B_{2}$ or its dual, but not both. We are going to characterize such groupoids in the next theorem, but first we need three lemmas. Let $\mathcal{A}$ denote the variety defined by $B_{1} \approx B_{2}$, i.e. $x(y(z u)) \approx x((y z) u)$.

Lemma 2.4. If $t_{1} \approx t_{2}$ is an identity that is true in every semigroup, then $\mathcal{A}$ satisfies $x t_{1} \approx x t_{2}$ (where $x$ is an arbitrary variable).

Proof. If $t_{1} \approx t_{2}$ holds in the variety of semigroups, then $t_{1}$ and $t_{2}$ are two bracketings of the same product. Therefore it suffices to prove that $\mathcal{A}$ satisfies $x \cdot B\left(x_{1}, \ldots, x_{n}\right) \approx x \cdot \overrightarrow{x_{1} \cdot \ldots \cdot x_{n}}$ for any bracketing $B\left(x_{1}, \ldots, x_{n}\right)$. We prove this by induction on $n$.

Repeatedly applying $x((y z) u) \approx x(y(z u))$ we can transform $x \cdot B\left(x_{1}, \ldots, x_{n}\right)$ to the form $x \cdot\left(x_{1} \cdot B^{\prime}\left(x_{2}, \ldots, x_{n}\right)\right)$. By the induction hypothesis we have that $x_{1}$. $B^{\prime}\left(x_{2}, \ldots, x_{n}\right) \approx x_{1} \cdot \overrightarrow{x_{2} \cdot \ldots \cdot x_{n}}=\overrightarrow{x_{1} \cdot \ldots \cdot x_{n}}$ holds in $\mathcal{A}$, hence $x \cdot B\left(x_{1}, \ldots, x_{n}\right) \approx$ $x \cdot \overrightarrow{x_{1} \cdot \ldots \cdot x_{n}}$ is true as well. (Note that we did nothing else but gave a proof for the general law of associativity, but we had to avoid implications of the form $p \approx q \Rightarrow p r \approx q r)$.

Lemma 2.5. Let $\mathcal{V}$ be a subvariety of $\mathcal{A}$, and let $\mathcal{W}$ be the intersection of $\mathcal{V}$ and the variety of semigroups. If an identity $t_{1} \approx t_{2}$ holds in $\mathcal{W}$, then $x t_{1} \approx x t_{2}$ holds in $\mathcal{V}$ (where $x$ is an arbitrary variable).

Proof. Let $\Theta_{\mathcal{V}}, \Theta_{\mathcal{W}}, \Theta_{\text {sgr }}$ denote the equational theories of $\mathcal{V}, \mathcal{W}$ and the variety of semigroups, respectively. These are fully invariant congruences of the free groupoid on countably many generators, and $\Theta_{\mathcal{W}}$ equals $\Theta_{\mathcal{V}} \vee \Theta_{\text {sgr }}$, i.e. the transitive closure of $\Theta_{\mathcal{V}} \cup \Theta_{\text {sgr }}$. Therefore, if $\mathcal{W}$ satisfies an identity $t_{1} \approx t_{2}$, then there are terms $p_{1}, \ldots, p_{n}$ such that $p_{1}=t_{1}, p_{n}=t_{2}$ and $p_{i} \approx p_{i+1}$ holds in $\mathcal{V}$ if $i$ is odd, and $p_{i} \approx p_{i+1}$ is a semigroup identity if $i$ is even. Then $x p_{i} \approx x p_{i+1}$ is true in $\mathcal{V}$ for every $i$ and any variable $x$. (For odd $i$ 's this is obvious; for even ones it follows from the previous lemma.) Now $x t_{1} \approx x t_{2}$ follows by transitivity.

The next lemma is based on the method used in the proof of Lemma 3.8 in [13], and is basically just a slight generalization of the situation considered there.

Lemma 2.6. Suppose that $\mathbb{A}$ is a groupoid with a minimal clone, and $M$ is a subset of $\mathrm{Clo}^{(2)}(\mathbb{A})$ containing the first projection and at least one nontrivial element, such that for all $f, g, h \in M$

(i) $f(g, h)=g$

(ii) $f\left(g, h^{d}\right)=f\left(g, e_{2}\right) \in M$.

Then $\mathbb{A}$ or its dual belongs to the variety $\mathcal{D}$ or $\mathcal{C}_{p}$ for some prime number $p$.

Proof. Let us recall that $e_{1}$ and $e_{2}$ are the first and second binary projection respectively (we can write $g^{d}$ as $g\left(e_{2}, e_{1}\right)$ with this notation). Note that $e_{2}=e_{1}^{d}$, hence (ii) means that $f\left(g, h^{d}\right)$ does not depend on $h$ (as long as $h \in M$ ). We have $e_{1} \in \bar{M}$, but $e_{2} \in M$ is impossible, because then (ii) would imply (with $f=e_{2}$ ) that $h^{d}=e_{2}$ for every $h \in M$, contradicting that $M$ has at least two elements. If $f \in M$ is nontrivial and $f^{d}$ also belongs to $M$, then we have $f\left(e_{1}, f^{d}\right)=e_{1}$ by (i) and $f\left(e_{1}, f^{d}\right)=f\left(e_{1}, e_{2}\right)=f$ by (ii), hence $f=e_{1}$, a contradiction. Thus $M$ and $M^{d}=\left\{f^{d}: f \in M\right\}$ are disjoint.

The operation $f * g=f\left(g, e_{2}\right)$ is associative in any clone, and $(M ; *)$ is a semigroup in virtue of (ii). The first projection is an identity element for $*$, hence $(M ; *)$ is a monoid. If $N$ is a submonoid of $M$, then $N \cup N^{d}$ is closed under binary compositions. In a minimal clone such a set must be either $\left\{e_{1}, e_{2}\right\}$ or the whole binary part of the clone. This fact together with the disjointness of $M$ and $M^{d}$ shows that $C l o^{(2)}(\mathbb{A})=M \cup M^{d}$, and the only submonoids of $M$ are $\left\{e_{1}\right\}$ and $M$ itself. Such a monoid is called minimal, and it was shown in Claim 3.11 of [13] that every minimal monoid is isomorphic to a two-element semilattice or a cyclic group of prime order. 
Suppose first that $(M ; *) \cong(\{0,1\} ; \vee)$ with $f_{0}$ and $f_{1}$ corresponding to 0 and 1 at this isomorphism. Then there are only four binary operations in $C l o(\mathbb{A})$, namely $e_{1}=f_{0}, e_{2}=f_{0}^{d}, f_{1}, f_{1}^{d}$ and we can suppose (after passing to the dual of $\mathbb{A}$ if necessary) that $f_{1}(x, y)=x y$, the basic operation in $\mathbb{A}$. By the above isomorphism we have $f_{1}=f_{1 \vee 1}=f_{1} * f_{1}=f_{1}\left(f_{1}, e_{2}\right)$, and this means that $x y \approx(x y) y$ holds in $\mathbb{A}$. Writing out (i) with $f=f_{1}, g=f_{1}, h=f_{0}$ and $f=f_{1}, g=f_{0}, h=f_{1}$ we get $f_{1}\left(f_{1}, f_{0}\right)=f_{1}$ and $f_{1}\left(f_{0}, f_{1}\right)=f_{0}$ implying that $\mathbb{A}$ satisfies the identities $(x y) x \approx$ $x y$ and $x(x y) \approx x$. Similarly we obtain $f_{1}\left(f_{0}, f_{1}^{d}\right)=f_{1}\left(f_{0}, e_{2}\right)$ and $f_{1}\left(f_{1}, f_{1}^{d}\right)=$ $f_{1}\left(f_{1}, e_{2}\right)$ as special cases of (ii), and they translate to the identities $x(y x) \approx x y$ and $(x y)(y x) \approx(x y) y$. All the identities in Proposition 1.3 are established, therefore $\mathbb{A} \in \mathcal{D}$ follows.

Now let us suppose that $(M ; *) \cong\left(\mathbb{Z}_{p} ;+\right)$ with $f_{i} \in M$ corresponding to $i \in \mathbb{Z}_{p}$. We have $f_{0}=e_{1}$ and we can suppose (after dualizing if necessary) that $f_{i}(x, y)=x y$ for some $i \in\{1, \ldots, p-1\}$. Since the automorphism group of $\mathbb{Z}_{p}$ acts transitively on $\{1, \ldots, p-1\}$, we can suppose without loss of generality that $f_{1}(x, y)=x y$. Then $f_{i+1}=f_{1} * f_{i}=f_{1}\left(f_{i}, e_{2}\right)$, thus $f_{i+1}(x, y)=f_{i}(x, y) \cdot y$, therefore $f_{i}(x, y)=x y^{i}$ and the binary part of $C l o(\mathbb{A})$ consists of the $2 p$ operations $f_{i}, f_{i}^{d}(i=0,1, \ldots, p-1)$. Similarly to the previous case, $\mathbb{F}_{2}(V(\mathbb{A}))$ can be determined: (i) implies $f_{i} \cdot f_{j}=$ $f_{1}\left(f_{i}, f_{j}\right)=f_{i}$, and (ii) implies $f_{i} \cdot f_{j}^{d}=f_{1}\left(f_{i}, f_{j}^{d}\right)=f_{1}\left(f_{i}, e_{2}\right)=f_{i+1}$; dualizing these we get $f_{i}^{d} \cdot f_{j}^{d}=f_{i}^{d}$ and $f_{i}^{d} \cdot f_{j}=f_{i+1}^{d}$. It is easy to check that $\mathbb{F}_{2}(V(\mathbb{A}))$ is a $p$-cyclic groupoid with a nontrivial clone (actually it is isomorphic to $\mathbb{F}_{2}\left(\mathcal{C}_{p}\right)$ ), hence $V(\mathbb{A})=\mathcal{C}_{p}$ by Proposition 1.2 .

Theorem 2.7. Let $\mathcal{V}$ be a subvariety of $\mathcal{A}$ having a minimal clone. Then $\mathcal{V}$ or its dual is a subvariety of $\mathcal{B}, \mathcal{C}_{p}, \mathcal{D}$ or the variety of rectangular bands.

Proof. Let $\mathcal{W}$ be the intersection of $\mathcal{V}$ and the variety of semigroups. Then $\mathcal{W}$ has a minimal or trivial clone, therefore it is a subvariety of the variety of left zero semigroups, right zero semigroups, rectangular bands, left regular bands or right regular bands (cf. [21, 30]). We treat these five cases separately.

Case 1. If $\mathcal{W}$ is the variety of left zero semigroups, then Lemma 2.5 shows that $\mathcal{V}$ satisfies $t_{1} x \approx t_{1} t$ for arbitrary terms $t_{1}, t$ if $x$ is the first variable of $t$. Specializing to $t=t_{1}$ we have that $\mathcal{V} \mid=t x \approx t t \approx t$, i.e. a $\mathcal{V}$-term does not change if we multiply it by its first variable from the right. Using these observations it is easy to check that $M=\left\{x, x y, x y^{2}, x y^{3}, \ldots\right\}$ satisfies the conditions of Lemma 2.6 for any $\mathbb{A} \in \mathcal{V}$ with a nontrivial clone (especially also for $\mathbb{F}_{\aleph_{0}}(\mathcal{V})$ ), and hence $\mathcal{V} \subseteq \mathcal{D}$ or $\mathcal{V}=\mathcal{C}_{p}$ for some prime $p$. (Note that $\mathcal{V}$ satisfies $x(y z) \approx x y$, therefore Lemma 4.3 of 32 ] could be used as well.)

Case 2. If $\mathcal{W}$ is the variety of right zero semigroups, then similarly to the previous case we have the identities $t_{1} x \approx t_{1} t$ and $t x \approx t$ in $\mathcal{V}$, where $x$ is the last variable of $t$. Now we can apply Lemma 2.6 with $\mathbb{A}=\mathbb{F}_{\aleph_{0}}(\mathcal{V})$ and $M=$ $\{x, \overleftarrow{x y x}, \overleftarrow{x y x y x}, \overleftarrow{x y x y x y x}, \ldots\}$ to show that $\mathcal{V} \subseteq \mathcal{D}$ or $\mathcal{V}=\mathcal{C}_{p}$ for some prime $p$ provided $\overleftarrow{x y x}$ is nontrivial in $\mathbb{F}_{\aleph_{0}}(\mathcal{V})$. If $(x y) x$ is a projection in $\mathbb{F}_{\aleph_{0}}(\mathcal{V})$, then $\mathcal{V} \models$ $(x y) x \approx x$ or $\mathcal{V} \models(x y) x \approx y$. The latter is impossible, since $x((x y) x) \approx x x \approx x$ holds in $\mathcal{V}$. Now we can write up the multiplication table of $\mathbb{F}_{2}(\mathcal{V})$.

\begin{tabular}{l|llll}
$\cdot$ & $x$ & $y$ & $x y$ & $y x$ \\
\hline$x$ & $x$ & $x y$ & $x y$ & $x$ \\
$y$ & $y x$ & $y$ & $y$ & $y x$ \\
$x y$ & $x$ & $x y$ & $x y$ & $x$ \\
$y x$ & $y x$ & $y$ & $y$ & $y x$
\end{tabular}

This is a semigroup in $\mathcal{V}$, but it is not a right zero semigroup, contradicting that $\mathcal{W}$ is the variety of right zero semigroups. (Actually this groupoid is isomorphic to the two-generated free rectangular band, hence Proposition 1.2 could be applied as well.)

Case 3. If $\mathcal{W}$ is the variety of rectangular bands, then $\mathcal{V}=\mathcal{W}$ by Proposition 1.2 
Case 4. Suppose now that $\mathcal{W}$ is a variety of left regular bands. Then $\mathcal{W}=t_{1} \approx t_{2}$ if $t_{1}$ and $t_{2}$ are binary terms such that both $x$ and $y$ appear in both terms, and they have the same first variable. Lemma 2.5 implies that $t t_{1} \approx t t_{2}$ holds in $\mathcal{V}$ for every term $t$, if $t_{1}$ and $t_{2}$ satisfy the above conditions. This allows us to perform the following computations in $\mathcal{V}$ with $g(x, y)=x(x y)$.

$$
\begin{aligned}
g(x, g(x, y)) & \approx x(x(x(x y))) \approx x(x y) \approx g(x, y) \\
g(x, g(y, x)) & \approx x(x(y(y x))) \approx x(x y) \approx g(x, y) \\
g(g(x, y), x) & \approx(x(x y))((x(x y)) x) \approx(x(x y))(x(x y)) \approx g(x, y) \\
g(g(x, y), y) & \approx(x(x y))((x(x y)) y) \approx(x(x y))(x(x y)) \approx g(x, y) \\
g(g(x, y), g(y, x)) & \approx(x(x y))((x(x y))(y(y x))) \approx(x(x y))(x(x y)) \approx g(x, y)
\end{aligned}
$$

These identities show that the subclone of $C l o(\mathcal{V})$ generated by $g$ contains at most four binary operations, namely $g, g^{d}$ and the two projections. If $g$ is nontrivial, then the minimality of the clone implies that $g(x, y)=x y$ or $g(y, x)=y x$. In the first case the above identities are just the axioms of $\mathcal{B}$, and in the second case they show that $\mathcal{V} \subseteq \mathcal{B}^{d}$. If $g$ is trivial, then $x(x y) \approx x$ holds in $\mathcal{V}$ (since $x(x y) \approx y$ is clearly impossible), and hence also in $\mathcal{W}$. Since $\mathcal{W}$ is a variety of bands, $\mathcal{W} \models x(x y) \approx x y$, and therefore it is the variety of left zero semigroups, and we have Case 1 .

Case 5. Finally, let $\mathcal{W}$ be a variety of right regular bands. Now $\mathcal{V} \models t t_{1} \approx t t_{2}$ whenever the last variable of the binary terms $t_{1}$ and $t_{2}$ is the same, and the same variables occur in them. Proceeding similarly to the previous case, we show that $[g]^{(2)}=\left\{e_{1}, e_{2}, g, g^{d}\right\}$ for $g(x, y)=x(y x)$. This is established by the following identities.

$$
\begin{aligned}
g(x, g(x, y)) & \approx x((x(y x)) x) \approx x(y x) \approx g(x, y) \\
g(x, g(y, x)) & \approx x((y(x y)) x) \approx x(y x) \approx g(x, y) \\
g(g(x, y), x) & \approx(x(y x))(x(x(y x))) \approx(x(y x))(x(y x)) \approx g(x, y) \\
g(g(x, y), y) & \approx(x(y x))(y(x(y x))) \approx(x(y x))(x(y x)) \approx g(x, y) \\
g(g(x, y), g(y, x)) & \approx(x(y x))((y(x y))(x(y x))) \approx(x(y x))(x(y x)) \approx g(x, y)
\end{aligned}
$$

If $g$ is nontrivial, then we have $\mathcal{V} \subseteq \mathcal{B}$ or $\mathcal{V} \subseteq \mathcal{B}^{d}$ just as in Case 4 . If $g$ is trivial, then it has to be a first projection, hence $x(y x) \approx x$ holds in $\mathcal{V}$. Right regular bands satisfy $x(y x) \approx y x$, hence $\mathcal{W} \models y x \approx x$, and we have Case 2 .

Now we are ready to prove the main result of this section, the characterization of groupoids with a minimal clone, that are almost semigroups in the 'spectral' sense.

Theorem 2.8. For a groupoid $\mathbb{A}$ the following two conditions are equivalent

(i) $\mathbb{A}$ has a minimal clone, and $1<s_{\mathbb{A}}(4)<5$;

(ii) $\mathbb{A}$ is not a semigroup, and $\mathbb{A}$ or its dual belongs to one of the varieties $\mathcal{B} \cap \mathcal{A}$, $\mathcal{C}_{p}$, or $\mathcal{D} \cap \mathcal{A}$.

If these conditions are fulfilled, then we have $s_{\mathbb{A}}(n)=2^{n-2}$ for $n \geq 2$.

Proof. First we show that (i) implies (ii). The considerations preceding Lemma 2.4 show that if $\mathbb{A}$ has a minimal clone, and $1<s_{\mathbb{A}}(4)<5$, then either $\mathbb{A}$ or its dual satisfies $x(y(z u)) \approx x((y z) u)$, i.e. $\mathbb{A} \in \mathcal{A}$ or $\mathbb{A} \in \mathcal{A}^{d}$. Applying Theorem 2.7 . we get that $\mathbb{A}$ or $\mathbb{A}^{d}$ belongs to $\mathcal{B}, \mathcal{C}_{p}$ or $\mathcal{D}$ (for some prime $p$ ). Thus we have to consider varieties of the from $\mathcal{V}_{1} \cap \mathcal{V}_{2}$, where $\mathcal{V}_{1}=\mathcal{A}$ or $\mathcal{V}_{1}=\mathcal{A}^{d}$, and $\mathcal{V}_{2} \in\left\{\mathcal{B}, \mathcal{C}_{p}, \mathcal{D}, \mathcal{B}^{d}, \mathcal{C}_{p}^{d}, \mathcal{D}^{d}: p\right.$ is a prime $\}$, but up to duality we have only six cases, because we may suppose that $\mathcal{V}_{2}=\mathcal{B}, \mathcal{C}_{p}$ or $\mathcal{D}$.

We show that if $\mathbb{A} \in \mathcal{V}_{2}$, and $a, b$ are elements of $\mathbb{A}$ such that $a x=b x$ holds for all $x \in \mathbb{A}$, then $a=b$. Letting $x=a$ and $x=b$ we see that $\{a, b\}$ is a right zero subsemigroup of $\mathbb{A}$. The identity $x(y x) \approx x y$ holds in $\mathcal{V}_{2}$ in all of the three cases, hence $a(b a)=a b$. Since $a$ and $b$ form a right zero semigroup we have $a(b a)=a$ 
and $a b=b$, thus $a=b$ as claimed. We see that $\mathcal{V}_{2} \cap \mathcal{A}^{d}$ is a variety of semigroups, because the defining identity of $\mathcal{A}^{d}$ is $((x y) z) u \approx(x(y z)) u$, and according to the previous observation this implies that $(x y) z \approx x(y z)$ holds in $\mathcal{V}_{2}$. Thus $\mathcal{V}_{1}=\mathcal{A}$, and we end up with the varieties of (ii). (Note that $\mathcal{C}_{p} \models x(y(z u)) \approx x y \approx x((y z) u)$, therefore $\mathcal{C}_{p} \cap \mathcal{A}=\mathcal{C}_{p}$.)

Now suppose that $\mathbb{A}$ (or its dual) belongs to one of the varieties mentioned in (ii) and $\mathbb{A}$ is not a semigroup. The clone of $\mathcal{B}, \mathcal{C}_{p}$ and $\mathcal{D}$ is minimal, thus the clone of $\mathbb{A}$ is minimal, too (note that $\mathbb{A}$ has a nontrivial clone, because it is not a semigroup). The other assertion of (i) will follow at once, if we prove that $s_{\mathbb{A}}(n)=2^{n-2}$. We will do this in two steps: first we show that $\mathbb{A} \in \mathcal{A}$ implies $s_{\mathbb{A}}(n) \leq 2^{n-2}$, and then we prove that $s_{\mathbb{A}}(n) \geq 2^{n-2}$ holds if we suppose in addition that $\mathbb{A} \in \mathcal{B}, \mathcal{C}_{p}$ or $\mathcal{D}$.

Let $B$ and $B^{\prime}$ be bracketings of the product $x_{1} \cdot \ldots \cdot x_{n}$. Lemma 2.4 implies that $\mathcal{A} \models B \approx B^{\prime}$ if $|l(B)|=\left|l\left(B^{\prime}\right)\right|$ and $\mathcal{A}=l(B) \approx l\left(B^{\prime}\right)$. Applying Lemma 2.4 again, we see that $|l(B)|=\left|l\left(B^{\prime}\right)\right|,\left|l^{2}(B)\right|=\left|l^{2}\left(B^{\prime}\right)\right|$ and $l^{2}(B) \approx l^{2}\left(B^{\prime}\right)$ is sufficient for $B \approx B^{\prime}$. Proceeding this way we arrive at left factors of size 1 (i.e. the single variable $x_{1}$ ) finally, and we see that if $\left|l^{i}(B)\right|=\left|l^{i}\left(B^{\prime}\right)\right|$ for all $i$ (where it makes sense), then $B \approx B^{\prime}$ holds in $\mathcal{A}$. Clearly, the numbers $\left|l^{i}(B)\right|$ (and $\left|l^{i}\left(B^{\prime}\right)\right|$ ) are strictly decreasing in $i$, therefore it is sufficient if the sets $\left\{\left|l^{i}(B)\right|: i=1,2, \ldots\right\}$ and $\left\{\left|l^{i}\left(B^{\prime}\right)\right|: i=1,2, \ldots\right\}$ coincide. They are subsets of $\{1,2, \ldots, n-1\}$, containing 1 , hence there are $2^{n-2}$ many choices for these sets. This shows that $s_{\mathbb{A}}(n) \leq 2^{n-2}$ for any $\mathbb{A} \in \mathcal{A}$.

Now let $\mathbb{A} \in \mathcal{A} \cap \mathcal{V}_{2}$, where $\mathcal{V}_{2} \in\left\{\mathcal{B}, \mathcal{C}_{p}, \mathcal{D}: p\right.$ is a prime $\}$, and let $B$ and $B^{\prime}$ be bracketings as before. Suppose that $\mathbb{A} \models B \approx B^{\prime}$, but $\left\{\left|l^{i}(B)\right|: i=1,2, \ldots\right\} \neq$ $\left\{\left|l^{i}\left(B^{\prime}\right)\right|: i=1,2, \ldots\right\}$, and let $i$ be the smallest value where $\left|l^{i}(B)\right|$ and $\left|l^{i}\left(B^{\prime}\right)\right|$ are different. Applying Lemma 2.4 and the observation made in the second paragraph of this proof (a certain right cancellation property) we can delete the right factors in the identity $B \approx B^{\prime}$, if they have the same size. Doing this $i-1$ times we arrive at bracketings whose left factors have different size, thus we may suppose that $i=1$, and we can also suppose that $\left|l^{1}(B)\right|<\left|l^{1}\left(B^{\prime}\right)\right|$. Let us substitute $x$ for the first $\left|l^{1}(B)\right|$ variables, $y$ for the next $\left|l^{1}\left(B^{\prime}\right)\right|-\left|l^{1}(B)\right|$ variables, and $z$ for the rest. Then $B$ becomes $(x \cdots x)(y \cdots y z \cdots z)$ (with some bracketing of the two products), and $B^{\prime}$ has the form $(x \cdots x y \cdots y)(z \cdots z)$. Thus $\mathbb{A}$ satisfies an identity of the form $(x \cdots x)(y \cdots y z \cdots z) \approx(x \cdots x y \cdots y)(z \cdots z)$ (with the same number of $x, y$ and $z$ on the two sides).

In $\mathcal{B}$ this identity reduces to $x(y z) \approx(x y) z$, showing that if $s_{\mathbb{A}}(n)<2^{n-2}$ for some $n$, then $\mathbb{A}$ is a semigroup. If $\mathcal{V}_{2}=\mathcal{C}_{p}$ or $\mathcal{D}$, then let us put $y=x$, then we have $\mathbb{A}=(x \cdots x)(x \cdots x z \cdots z) \approx(x \cdots x x \cdots x)(z \cdots z)$. The right hand side is clearly $x z$, and on the left hand side the bracketing of the factor $(x \cdots x z \cdots z)$ is irrelevant according to Lemma 2.4 . Thus $\mathbb{A} \models x(x z) \approx x z$, and since $x(x z) \approx x$ holds in $\mathcal{C}_{p}$ and $\mathcal{D}$ we see that $\mathbb{A}$ is a left zero semigroup. We have proved that the associative spectrum of a groupoid in any one of the varieties mentioned in (ii) is either $(1,1,1,1, \ldots)$ or $(1,2,4,8, \ldots)$, and this completes the proof of the theorem.

Remark. Each of the varieties $\mathcal{B} \cap \mathcal{A}, \mathcal{C}_{p}$ and $\mathcal{D} \cap \mathcal{A}$ contain groupoids with a nonassociative operation. For $\mathcal{C}_{p}$ it is clear, because the only $p$-cyclic groupoids that are semigroups are the left zero semigroups. The two-generated free algebra of $\mathcal{D}$ is not a semigroup, and satisfies $x(y(z u)) \approx x((y z) u)$, hence belongs to $\mathcal{D} \cap \mathcal{A}$. (See the multiplication table in the proof of Proposition 1.3.) Let us now construct some nonassociative algebras in $\mathcal{B} \cap \mathcal{A}$.

Let $\mathbb{S}=(S ; \vee)$ be a semilattice, and let $C$ be the set of finite chains in $\mathbb{S}$. We define a multiplication in $C$ by the following formula (note that if $b_{l} \leq a_{k}$, then the right hand side is the same as the first factor on the left hand side).

$$
\left(a_{1}<a_{2}<\cdots<a_{k}\right) \cdot\left(b_{1}<b_{2}<\cdots<b_{l}\right)=\left(a_{1}<a_{2}<\cdots<a_{k} \leq a_{k} \vee b_{l}\right)
$$


For $\mathbf{a}=\left(a_{1}<a_{2}<\cdots<a_{k}\right), \mathbf{b}=\left(b_{1}<b_{2}<\cdots<b_{l}\right)$ and $\mathbf{c}=\left(c_{1}<c_{2}<\cdots<c_{m}\right)$ we have $(\mathbf{a} \cdot \mathbf{b}) \cdot \mathbf{c}=\left(a_{1}<a_{2}<\cdots<a_{k} \leq a_{k} \vee b_{l} \leq a_{k} \vee b_{l} \vee c_{m}\right)$ and $\mathbf{a} \cdot(\mathbf{b} \cdot \mathbf{c})=$ $\left(a_{1}<a_{2}<\cdots<a_{k} \leq a_{k} \vee b_{l} \vee c_{m}\right)$. Since the top element of both chains is $a_{k} \vee$ $b_{l} \vee c_{m}$, right multiplication by $(\mathbf{a} \cdot \mathbf{b}) \cdot \mathbf{c}$ is the same as right multiplication by $\mathbf{a} \cdot(\mathbf{b} \cdot \mathbf{c})$, hence $\mathbb{C}=(C ; \cdot)$ satisfies $x(y(z u)) \approx x((y z) u)$. It is not hard to check, that the defining identities of $\mathcal{B}$ also hold in $\mathbb{C}$, hence $\mathbb{C} \in \mathcal{B} \cap \mathcal{A}$. If the height of $\mathbb{S}$ is at least three, i.e. there is a chain of length three, then $\mathbb{C}$ is not a semigroup. Indeed, if $a<b<c$, then $(a \cdot b) \cdot c=(a<b<c) \neq(a<c)=a \cdot(b \cdot c)$.

Remark. The variety $\mathcal{D} \cap \mathcal{A}$ was defined by an infinite set of identities, but it has a finite basis, namely $x x \approx x, x(y z) \approx x y,(x y) y \approx x y$. Indeed, it is quite straightforward to check that any algebra satisfying these identities belongs to $\mathcal{D} \cap \mathcal{A}$. Conversely, if $\mathbb{A} \in \mathcal{D} \cap \mathcal{A}$, then $\mathbb{A} \models x(y z) \approx x((y y) z) \approx x(y(y z)) \approx x y$, and $\mathbb{A}$ also satisfies $x x \approx x$ and $(x y) y \approx x y$ as they are among the defining identities of $\mathcal{D}$. This latter axiomatization of $\mathcal{D} \cap \mathcal{A}$ resembles to the definition of $p$-cyclic groupoids. It is an interesting fact that every groupoid that has a minimal clone and satisfies $x(y z) \approx x y$ belongs to one of the varieties $\mathcal{C}_{p}$ or $\mathcal{D} \cap \mathcal{A}$ (cf. Lemma 4.3 of 32$])$.

\section{SzÁsz-HÁJEK GROUPOIDS With A MINIMAL CLONE}

Another way to measure associativity is to count the number of nonassociative triples in the groupoid; this number (or cardinal, in the infinite case) is called the index of nonassociativity, and is denoted by $n s$. Formally, we have $n s(\mathbb{A})=$ $\left|\left\{(a, b, c) \in A^{3}:(a b) c \neq a(b c)\right\}\right|$. This notion has been studied by several authors 2, 3, 7, 14, 27]. Clearly $\mathbb{A}$ is a semigroup iff $n s(\mathbb{A})=0$, and it is natural to say that the multiplication of $\mathbb{A}$ is almost associative, if $n s(\mathbb{A})=1$. Such groupoids are called Szász-Hájek groupoids (SH-groupoids for short). SH-groupoids were investigated in [9, 10] and 16, 17, 18, 19] in much detail. Following the terminology of these papers, we say that an SH-groupoid is of type $(a, b, c)$, if its only nonassociative triple is $(a, b, c) \in A^{3}$ and $a \neq b \neq c \neq a$. Types $(a, a, a),(a, b, a),(a, a, b)$ and $(a, b, b)$ are defined analogously. (Note that by saying e.g. that $\mathbb{A}$ is an SH-groupoid of type $(a, b, c)$ we mean not only that the components of the unique nonassociative triple are pairwise distinct, but implicitly we assume that these components are denoted by $a, b$ and $c$ respectively.) Let us recall a result from [16] (Proposition 1.2(i)).

Proposition 3.1. If $\mathbb{A}$ is an SH-groupoid, and $(a, b, c)$ is the unique nonassociative triple, then $x y=a(x y=b, x y=c)$ implies $x=a(x=b, x=c)$ or $y=a$ $(y=b, y=c)$ for all $x, y \in A$.

Proof. Suppose that $x y=a$, but $x \neq a \neq y$. Since $x \neq a$, we have $(x, y, b c) \neq$ $(a, b, c)$, hence $(x, y, b c)$ is an associative triple: $(x y)(b c)=x(y(b c))$. Now $y \neq a$ implies that $(y, b, c) \neq(a, b, c)$, so $x(y(b c))=x((y b) c)$. Similarly $x((y b) c)=$ $(x(y b)) c=((x y) b) c$, because $x \neq a$. We have obtained that $(x y)(b c)=((x y) b) c$, thus $(x y, b, c)=(a, b, c)$ is an associative triple, which is a contradiction. The other two assertions can be proved similarly.

Clearly, a subgroupoid of an $\mathrm{SH}$-groupoid $\mathbb{A}$ with nonassociative triple $(a, b, c)$ is an SH-groupoid or a semigroup, depending on whether it contains $a, b$ and $c$ or not. Specially, $\mathbb{A}$ is generated by $\{a, b, c\}$ iff all proper subgroupoids of $\mathbb{A}$ are semigroups. Such a groupoid is called a minimal SH-groupoid. In [16, 17, 18, 19] the project of characterizing minimal SH-groupoids was begun, but completed only for the type $(a, a, a)$. In Theorem 3.3 we prove that $\mathrm{SH}$-groupoids having a minimal clone belong to the varieties $\mathcal{B}$ or $\mathcal{B}^{d}$, and in Theorem 3.4 we give a complete list of minimal SH-groupoids with a minimal clone up to isomorphism. We need the following lemma before we state and prove the main result.

Lemma 3.2. If an SH-groupoid has a minimal clone, then it is of type $(a, b, c)$. 
Proof. Let $\mathbb{A}$ be an SH-groupoid with a minimal clone. Then $\mathbb{A}$ is idempotent, hence it cannot be of type $(a, a, a)$. If it is of type $(a, b, a)$, then the subgroupoid generated by $a$ and $b$ is a minimal $\mathrm{SH}$-groupoid of type $(a, b, a)$ with a minimal clone. The description of minimal SH-groupoids of type $(a, b, a)$ given in [17] is not complete, but it covers the idempotent case (subtypes $(\alpha)$ and $(\beta))$. There are four idempotent minimal SH-groupoids of type $(a, b, a)$ up to isomorphism: the following two groupoids and their duals (the second groupoid is a factor of the first one).

\begin{tabular}{|c|c|c|c|c|c|c|c|}
\hline · & $a$ & $b$ & $d$ & $e$ & - & $a$ & $b$ \\
\hline$a$ & $a$ & $a$ & $e$ & $e$ & $a$ & $a$ & $a$ \\
\hline$b$ & $d$ & $b$ & $d$ & $d$ & $b$ & $d$ & $b$ \\
\hline$d$ & $d$ & $d$ & $d$ & $d$ & $d$ & $d$ & $d$ \\
\hline$e$ & $e$ & $e$ & $e$ & $e$ & & & \\
\hline
\end{tabular}

In both cases the operation $g(x, y)=x(y x)$ is nontrivial, and preserves the equivalence relation corresponding to the partition whose only nontrivial block is $\{b, d\}$, but the basic operation $f(x, y)=x y$ does not preserve this relation. This shows that $f \notin[g]$, hence the clone is not minimal.

Suppose now that $\mathbb{A}$ is of type $(a, a, b)$. From the computations in 18 , it follows that $d=b a=b$ (combine Lemmas 1.5, 1.6, 2.4 and 2.19), therefore the subgroupoid generated by $a$ and $b$ is a minimal SH-groupoid of type $(a, a, b)$ and of subtype $(\varepsilon)$. Up to isomorphism there is only one such groupoid, namely the following one.

\begin{tabular}{l|llll}
$\cdot$ & $a$ & $b$ & $c$ & $e$ \\
\hline$a$ & $a$ & $c$ & $e$ & $e$ \\
$b$ & $b$ & $b$ & $b$ & $b$ \\
$c$ & $c$ & $c$ & $c$ & $c$ \\
$e$ & $e$ & $e$ & $e$ & $e$
\end{tabular}

The clone of this groupoid is not minimal, because $x(x y)$ is a nontrivial operation preserving the set $\{a, b, e\}$, while the basic operation $x y$ does not preserve this set.

Dually, the type $(a, b, b)$ is not possible either, thus we can conclude that an $\mathrm{SH}$-groupoid with a minimal clone has to be of type $(a, b, c)$.

Theorem 3.3. For a Szász-Hájek groupoid $\mathbb{A}$ the following two conditions are equivalent.

(i) $\mathbb{A}$ has a minimal clone;

(ii) $\mathbb{A}$ or its dual belongs to the variety $\mathcal{B}$.

Proof. It is clear that (ii) implies (i), since $\mathcal{B}$ has a minimal clone. For the other direction let us suppose that $\mathbb{A}$ is an $\mathrm{SH}$-groupoid with a minimal clone. As we have seen in the previous lemma, $\mathbb{A}$ is of type $(a, b, c)$. Therefore $(x, x, y)$ is an associative triple for all $x, y \in A$, hence $\mathbb{A} \models x(x y) \approx x y$ by idempotence. Similarly, we obtain $\mathbb{A} \models(x y) y \approx x y$ and $\mathbb{A} \models x(y x) \approx(x y) x$. Proposition 3.1 shows that $(x, y, x y)$ is an associative triple for all $x, y \in A$, because $x=a, y=b, x y=c$ is impossible. Thus $\mathbb{A} \mid=x(y(x y)) \approx(x y)(x y) \approx x y$. By another application of Proposition 3.1 we can see that $(x y, y, x) \neq(a, b, c)$, so $(x y)(y x) \approx((x y) y) x \approx(x y) x$ holds in $\mathbb{A}$.

The identities derived so far are almost sufficient to fill out the multiplication table of the two-generated free algebra in the variety generated by $\mathbb{A}$ (see the table below). The only entries that are not determined yet are $(x y x)(y x y)$ and $(y x y)(x y x)$. In order to compute these, let us observe that $(x y x, y x, y)$ is always an associative triple, because $y x=b$ and $y=c$ implies $x=b$ by Proposition 3.1, but then $x y x=b b=b \neq a$. Therefore $\mathbb{A} \models(x y x)(y x y) \approx((x y x)(y x)) y \approx(x y x) y \approx x y$. 


\begin{tabular}{l|llllll}
$\cdot$ & $x$ & $y$ & $x y$ & $y x$ & $x y x$ & $y x y$ \\
\hline$x$ & $x$ & $x y$ & $x y$ & $x y x$ & $x y x$ & $x y$ \\
$y$ & $y x$ & $y$ & $y x y$ & $y x$ & $y x$ & $y x y$ \\
$x y$ & $x y x$ & $x y$ & $x y$ & $x y x$ & $x y x$ & $x y$ \\
$y x$ & $y x$ & $y x y$ & $y x y$ & $y x$ & $y x$ & $y x y$ \\
$x y x$ & $x y x$ & $x y$ & $x y$ & $x y x$ & $x y x$ & $x y$ \\
$y x y$ & $y x$ & $y x y$ & $y x y$ & $y x$ & $y x$ & $y x y$
\end{tabular}

We see that the binary part of $C l o(\mathbb{A})$ contains at most six operations (some of the six elements in the table may coincide). In [20] we can find the complete description of minimal clones with at most six binary operations, so we could finish the proof by simply examining the list of clones given there.

Another way is to observe that for $g(x, y)=x y x$ the binary part of $[g]$ is $\left\{e_{1}, e_{2}, g, g^{d}\right\}$. If $g$ is a nontrivial operation, then $[g]=C l o(\mathbb{A})$, hence $\mathbb{A}$ satisfies $x y x \approx x y$ or $x y x \approx y x$, and then the defining identities of $\mathcal{B}$ or $\mathcal{B}^{d}$ can be read from the above multiplication table. If $g$ is trivial, then $\mathbb{A} \models x y x \approx x$, because $x y x \approx y$ would imply $x y \approx(x y x) y \approx y y \approx y$. In this case $\mathbb{F}_{2}(V(\mathbb{A}))$ is a rectangular band (we get the same multiplication table as in Case 2 of the proof of Theorem 2.7 , hence $\mathbb{A}$ is a rectangular band by Proposition 1.2 , contradicting that $\mathbb{A}$ is an SH-groupoid.

Finally we describe minimal $\mathrm{SH}$-groupoids in the varieties $\mathcal{B}$ and $\mathcal{B}^{d}$ up to isomorphism.

Theorem 3.4. Every minimal SH-groupoid having a minimal clone is isomorphic or dually isomorphic to one of the groupoids $\mathbb{G}_{1}, \ldots, \mathbb{G}_{10}$ (see the multiplication tables in the proof).

Proof. Let $\mathbb{A}$ be a minimal $\mathrm{SH}$-groupoid with a minimal clone. Then $\mathbb{A}$ is of type $(a, b, c)$, and up to duality we may suppose that $\mathbb{A}$ belongs to the variety $\mathcal{B}$. Following the notation of [19] we set $d=a b, e=b c, f=a(b c)=a e$ and $g=(a b) c=d c$. Some of these elements may coincide, but $a, b, c$ are pairwise distinct and $f \neq g$. Since $\mathbb{A}$ is idempotent, we have $d=a$ or $e=c$ by Lemma 1.7 of [19]. If $d=a$, then $b a=b$ or $b a=a($ Lemma 1.9 (iii)); if $e=c$, then $c b=b$ or $c b=c$ (Lemma 1.9 (iv)). Thus we have four cases, and we will deal with them separately.

Case 1. $d=a b=a$ and $b a=b$

We have $g=d c=a c=c$ by Lemma 1.4 (ii) of [19], and then $c a=c(c a)=$ $(a c)(c a)=a c=c$ follows applying the defining identities of $\mathcal{B}$. Some other products may be computed with the help of these identities, for example $b e=b(b c)=b c=e$ and $e b=(b c) b=b c=e$. For others, we can use the fact that $(a, b, c)$ is the only nonassociative triple, e.g.: $c b=(c a) b=c(a b)=c a=c$, and $b f=b(a e)=(b a) e=$ $b e=e$.

We can fill out the multiplication table this way except for the entry $f c$. Here we have two possibilities. If $f \neq a$ or $e \neq b$, then $(f, e, c) \neq(a, b, c)$, therefore $f c=(f e) c=f(e c)=f e=f$, and we get the groupoid $\mathbb{G}_{1}$. If $f=a$ and $e=b$, then $f c=a c=c$, and we arrive at the groupoid $\mathbb{G}_{3}$. In both cases we have to consider the possibility that some of the elements (denoted by different letters so far) coincide. This amounts to forming factor groupoids, but only with respect to congruences where $f$ and $g$ belong to different congruence classes (otherwise the factor groupoid would be a semigroup). There is no such congruence on $\mathbb{G}_{3}$, while $\mathbb{G}_{1}$ has exactly one nontrivial congruence not collapsing $f$ and $g(=c)$; its classes 
are $\{a\},\{b\},\{c\},\{e, f\}$, and the corresponding factor groupoid is $\mathbb{G}_{2}$.

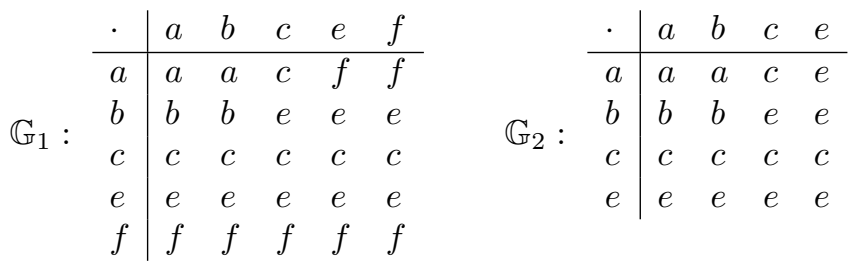

$$
\begin{aligned}
& \mathbb{G}_{3}: \begin{array}{l|lll}
\cdot & a & b & c \\
\hline a & a & a & c \\
b & b & b & b \\
c & c & c & c
\end{array}
\end{aligned}
$$

Case 2. $d=a b=a$ and $b a=a$

Let us start again with the product $c a$. We claim that $(a, b, c a)$ is a nonassociative triple. Indeed, $(a b)(c a)=a(c a)=a c=(a b) c$, while $a(b(c a))=a((b c) a)=$ $a(e a)=a e=a(b c)$. Since the only nonassociative triple is $(a, b, c)$, we can conclude that $c a=c$. Then $c b=(c a) b=c(a b)=c a=c$, and the rest of the multiplication table can be filled out without any difficulty (we will not have to deal with a situation like that of $f c$ in the previous case). We get the groupoid $\mathbb{G}_{4}$, and the only possible coincidence between the six elements is $e=f$; this yields $\mathbb{G}_{5}$.

$\mathbb{G}_{4}:$\begin{tabular}{l|llllll}
$\cdot$ & $a$ & $b$ & $c$ & $e$ & $f$ & $g$ \\
\hline$a$ & $a$ & $a$ & $g$ & $f$ & $f$ & $g$ \\
$b$ & $a$ & $b$ & $e$ & $e$ & $f$ & $g$ \\
$c$ & $c$ & $c$ & $c$ & $c$ & $c$ & $c$ \\
$e$ & $e$ & $e$ & $e$ & $e$ & $e$ & $e$ \\
$f$ & $f$ & $f$ & $f$ & $f$ & $f$ & $f$ \\
$g$ & $g$ & $g$ & $g$ & $g$ & $g$ & $g$
\end{tabular}

$\mathbb{G}_{5}:$\begin{tabular}{c|ccccc}
$\cdot$ & $a$ & $b$ & $c$ & $e$ & $g$ \\
\hline$a$ & $a$ & $a$ & $g$ & $e$ & $g$ \\
$b$ & $a$ & $b$ & $e$ & $e$ & $g$ \\
$c$ & $c$ & $c$ & $c$ & $c$ & $c$ \\
$e$ & $e$ & $e$ & $e$ & $e$ & $e$ \\
$g$ & $g$ & $g$ & $g$ & $g$ & $g$
\end{tabular}

Case 3. $e=b c=c$ and $c b=b$

As the following computation shows, this case is not possible, because the identities of $\mathcal{B}$ imply that $\mathbb{A}$ is a semigroup. (We have indicated where we used the axioms of $\mathcal{B}$ and the Szász-Hájek property.)

$$
\begin{aligned}
& g=d c \stackrel{\mathcal{B}}{=}(d c) c=(d c) e \stackrel{S H}{=} d(c e)=d(c(b c)) \\
& \stackrel{\mathcal{B}}{=} d(c b)=(a b)(c b) \stackrel{S H}{=} a(b(c b)) \stackrel{\mathcal{B}}{=} a(b c)=f
\end{aligned}
$$

Case 4. $e=b c=c$ and $c b=c$

We prove that $c d=c$ by showing that $(a, b, c d)$ is a nonassociative triple. Indeed, $(a b)(c d)=d(c d)=d c=g$, while $a(b(c d))=f$ can be derived in the following way.

$$
\begin{aligned}
a(b(c d)) \stackrel{S H}{=} a((b c) d)=a(c d) & \stackrel{S H}{=}(a c) d=(a c)(a b) \\
& \stackrel{S H}{=}((a c) a) b \stackrel{\underline{\mathcal{B}}}{=}(a c) b \stackrel{S H}{=} a(c b)=a c=a(b c)=f
\end{aligned}
$$

Now we can compute that $c a=(c d) a=c(d a)=c((a b) a)=c(a b)=c d=c$, and the rest of the multiplication table of $\mathbb{G}_{6}$ is not hard to fill out (we set $h=b a$ and $i=b f$ ). The only entries whose calculation is not straightforward are $a g$, ai and $d i$. Since $f \neq g$, at least one of these two elements is different from $c$, hence $(a, d, f)$ or $(a, d, g)$ is an associative triple (even if $d=b)$. Therefore we have either $a g=a(d f)=(a d) f=d f=g$, or $a g=a(d g)=(a d) g=d g=g$ (after computing $d f=d g=g$ and $a d=d$, which is easy). Writing $a i$ either as $a(b f)$ or $a(b g)$ and $d i$ as $d(b f)$ or $d(b g)$ we get by a similar argument that $a i=g$ and $d i=g$. 
There are four congruences of $\mathbb{G}_{6}$ that do not collapse $f$ and $g$, the corresponding factor groupoids are $\mathbb{G}_{7}, \mathbb{G}_{8}, \mathbb{G}_{9}$ and $\mathbb{G}_{10}$.

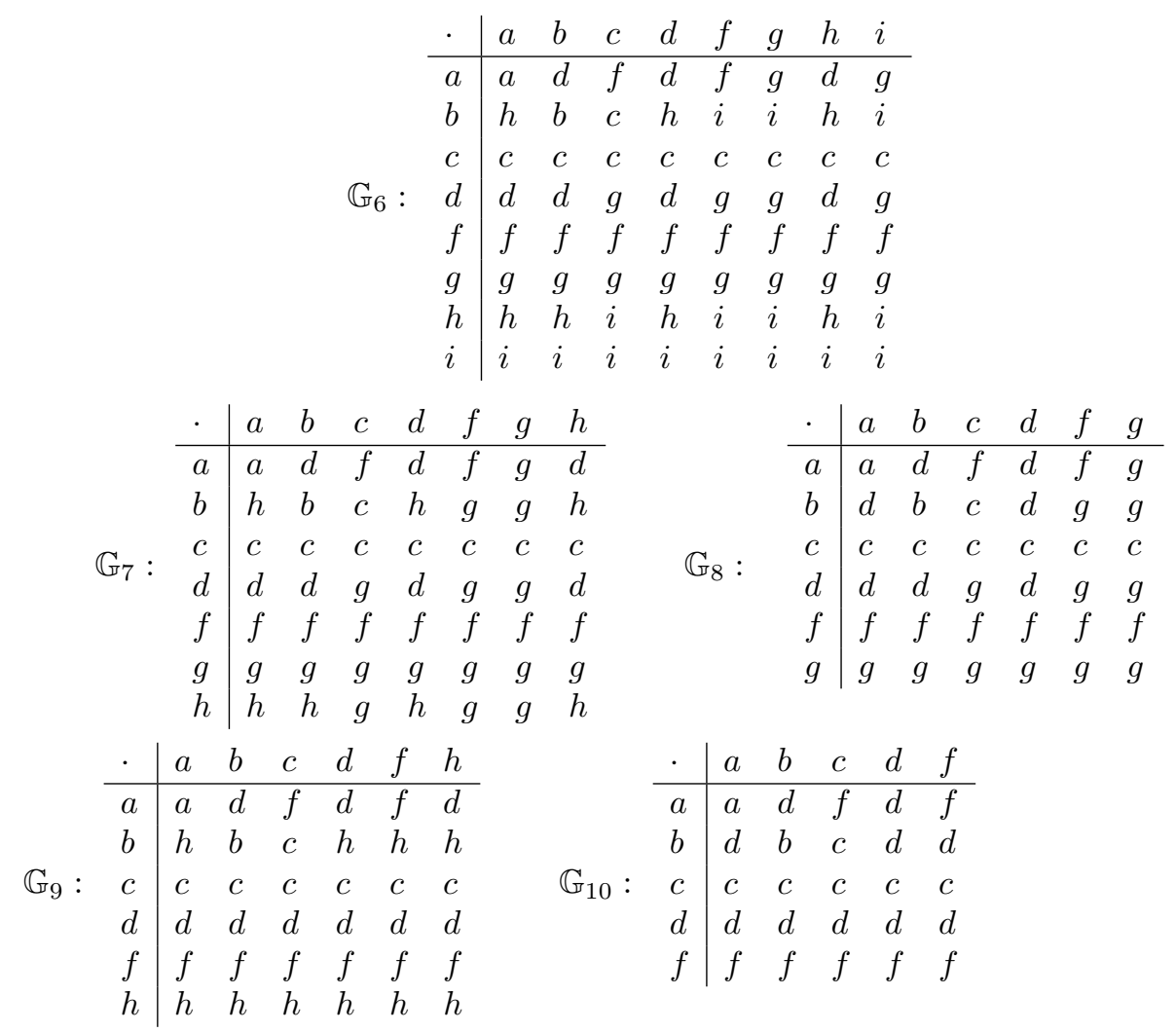

Remark. Let us mention that there is a third possibility to measure associativity with the help of the Hamming distance of multiplication tables. This yields the notion of the semigroup distance of a groupoid. Groupoids with small semigroup distance, and connections between the semigroup distance and the index of nonassociativity were studied in [15].

The different ways of measuring associativity do not seem to be closely related. For example, the groupoid $\mathbb{G}_{3}$ is an SH-groupoid, with the largest possible associative spectrum: $s_{\mathbb{G}_{3}}(n)=C_{n-1}$ for every $n$. (For the proof of the latter fact see 5.1 in [6]; $\mathbb{G}_{3}$ is isomorphic to the groupoid with number 17 there.)

Therefore it is not surprising that the class of groupoids found in Theorem 2.8 is disjoint from the class described in Theorem 3.3 , i.e. there is no groupoid with a minimal clone that is almost associative in both the 'spectral' and the 'index' sense. Indeed, if $\mathbb{A}$ satisfies the conditions of both theorems, then $\mathbb{A}$ (or its dual) satisfies $x(y(z u)) \approx x((y z) u)$ by the considerations preceding Lemma 2.4 and $\mathbb{A}$ (or its dual) contains a subgroupoid isomorphic to one of the groupoids $\mathbb{G}_{1}, \ldots, \mathbb{G}_{10}$ by Theorem 3.4. However, this is impossible, because neither of these ten groupoids and neither of their duals satisfy $x(y(z u)) \approx x((y z) u)$ as it can be seen from their multiplication tables (let $x=a, y=a, z=b, u=c$ for $\mathbb{G}_{1}, \ldots, \mathbb{G}_{10}$ and $x=a, y=c, z=b, u=a$ for their duals).

\section{REFERENCES}

[1] V. G. Bodnarčuk, L. A. Kalužnin, V. N. Kotov, B. A. Romov, Galois theory for Post algebras I-II, Kibernetika (Kiev) 3 (1969), 1-10; 5 (1969), 1-9. (Russian)

[2] A. C. Climescu, Études sur la théorie des systèmes multiplicatifs uniformes I. L'indice de non-associativité, Bull. École Polytech. Jassy 2 (1947), 347-371. (French)

[3] A. C. Climescu, L'indépendance des conditions d'associativité, Bull. Inst. Polytech. Jassy 1 (1955), 1-9. (Romanian) 
[4] B. Csákány, All minimal clones on the three-element set, Acta Cybernet. 6 (1983), no. 3, 227-238.

[5] B. Csákány, On conservative minimal operations, Lectures in Universal Algebra (Szeged, 1983), Colloq. Math. Soc. János Bolyai, 43, North-Holland, Amsterdam, 1986, 49-60.

[6] B. Csákány, T. Waldhauser, Associative spectra of binary operations, Mult.-Valued Log. 5 (2000), no. 3, 175-200.

[7] A. Drápal, T. Kepka, Sets of associative triples, Europ. J. Combinatorics 6 (1985), 227-231.

[8] D. Geiger, Closed systems and functions of predicates, Pacific J. Math. 27 (1968), 95-100.

[9] P. Hájek, Die Szászschen Gruppoide, Mat.-Fys. Časopis Sloven. Akad. Vied 15 (1965) no. 1., 15-42. (German)

[10] P. Hájek, Berichtigung zu meiner arbeit "Die Szászschen Gruppoide", Mat.-Fys. Casopis Sloven. Akad. Vied 15 (1965) no. 4., 331. (German)

[11] J. Ježek, R. W. Quackenbush, Minimal clones of conservative functions, Internat. J. Algebra Comput. 5 (1995), no. 6, 615-630.

[12] K. A. Kearnes, Minimal clones with abelian representations, Acta Sci. Math. (Szeged) 61 (1995), no. 1-4, 59-76.

[13] K. A. Kearnes, Á. Szendrei, The classification of commutative minimal clones, Discuss. Math. Algebra Stochastic Methods 19 (1999), no. 1, 147-178.

[14] T. Kepka, M. Trch, Groupoids and the associative law I. (Associative triples), Acta Univ. Carol. Math. Phys. 33 (1992), no. 1., 69-86.

[15] T. Kepka, M. Trch, Groupoids and the associative law II. (Groupoids with small semigroup distance), Acta Univ. Carol. Math. Phys. 34 (1993), no. 1., 67-83.

[16] T. Kepka, M. Trch, Groupoids and the associative law III. (Szász-Hájek groupoids), Acta Univ. Carol. Math. Phys. 36 (1995), no. 1., 17-30.

[17] T. Kepka, M. Trch, Groupoids and the associative law IV. (Szász-Hájek groupoids of type $(a, b, a))$, Acta Univ. Carol. Math. Phys. 35 (1994), no. 1., 31-42.

[18] T. Kepka, M. Trch, Groupoids and the associative law V. (Szász-Hájek groupoids of type $(a, a, b))$, Acta Univ. Carol. Math. Phys. 36 (1995), no. 1., 31-44.

[19] T. Kepka, M. Trch, Groupoids and the associative law VI. (Szász-Hájek groupoids of type $(a, b, c))$, Acta Univ. Carol. Math. Phys. 38 (1997), no. 1., 13-22.

[20] L. Lévai, P. P. Pálfy, On binary minimal clones, Acta Cybernet. 12 (1996), no. 3, $279-294$.

[21] P. P. Pálfy, Minimal clones, Preprint of the Math. Inst. Hungarian Acad. Sci. 27/1984.

[22] J. Płonka, On groups in which idempotent reducts form a chain, Colloq. Math. 29 (1974), $87-91$.

[23] J. Płonka, On k-cyclic groupoids, Math. Japon. 30 (1985), no. 3, 371-382.

[24] E. Post, The two-valued iterative systems of mathematical logic, Annals of Mathematics Studies, no. 5, Princeton University Press, Princeton, 1941.

[25] I. G. Rosenberg, Minimal clones I. The five types, Lectures in Universal Algebra (Szeged, 1983), Colloq. Math. Soc. János Bolyai, 43, North-Holland, Amsterdam, 1986, 405-427.

[26] N. J. A. Sloane, The On-Line Encyclopedia of Integer Sequences, http://www.research.att.com/ njas/sequences, 2005.

[27] G. Szász, Die Unabhängigkeit der Assoziativitätsbedingungen, Acta Sci. Math. (Szeged) 15 (1953), 20-28. (German)

[28] B. Szczepara, Minimal clones generated by groupoids, Ph.D. Thesis, Université de Montréal, 1995.

[29] Á. Szendrei, Clones in Universal Algebra, Séminaire de Mathématiques Supérieures, 99, Presses de L'Université de Montréal, 1986.

[30] M. B. Szendrei On closed sets of term functions on bands, Semigroups (Proc. Conf., Math. Res. Inst., Oberwolfach, 1978), pp. 156-181, Lecture Notes in Math., 855, Springer, Berlin, 1981.

[31] T. Waldhauser, Minimal clones generated by majority operations, Algebra Universalis 44 (2000), no. 1-2, 15-26.

[32] T. Waldhauser, Minimal clones with weakly abelian representations, Acta Sci. Math. (Szeged) 69 (2003), no. 3-4, 505-521.

Bolyai Institute, University of Szeged, Aradi vértanúk tere 1, H6720, Szeged, HunGARY

E-mail address: twaldha@math.u-szeged.hu 